\title{
Raman forward scattering of chirped laser pulses
}

\author{
C. B. Schroeder, E. Esarey, B. A. Shadwick, and W. P. Leemans \\ Lawrence Berkeley National Laboratory, \\ University of California, Berkeley, California 94720
}

(Dated: December 5, 2002)

\begin{abstract}
Raman scattering of a high-intensity, short-duration, frequency-chirped laser pulse propagating in an underdense plasma is examined. The growth of the direct forward scattered light is calculated for a laser pulse with a linear frequency chirp in various spatiotemporal regimes. This includes a previously undescribed regime of strongly-coupled four-wave nonresonant interaction, which is important for relativistic laser intensities. In all regimes of forward scattering, it is shown that the growth rate increases (decreases) for positive (negative) frequency chirp. The effect of chirp on the growth rate is relatively minor, i.e., a few percent chirp yields few percent changes in the growth rates. Numerical solutions based on a fully nonlinear cold Maxwell-fluid model are presented which confirm analytical predictions. Relation of these results to recent experiments is discussed.
\end{abstract}

PACS numbers: 52.38.-r, 52.38.Bv, 52.38.Kd 


\section{INTRODUCTION}

High-intensity short-pulse laser-plasma interactions are of much current interest because of their application to laser-plasma accelerators ${ }^{1}$, laser-plasma-based harmonic generation ${ }^{2}$, $\mathrm{x}$-ray lasers $^{3}$, and laser-driven inertial confinement fusion schemes ${ }^{4}$. A basic phenomenon in laser-plasma interactions is Raman scattering. The Raman scattering instability ${ }^{5}$ is the resonant decay in a plasma of incident light, with frequency and wave number $\left(\omega_{0}, k_{0}\right)$, into a plasma wave $(\omega, k)$ and scattered light $\left(\omega_{0} \pm \omega, k_{0} \pm k\right)$. Physically, the Raman instability occurs due to the beating of the incident and scattered light, producing a ponderomotive force which generates a plasma density modulation at or near the plasma frequency $\omega_{p}=\left(4 \pi e^{2} n_{0} / m\right)^{1 / 2}$, where $e$ and $m$ are the electronic charge and mass and $n_{0}$ is the equilibrium electron plasma density. The plasma density (index of refraction) modulation causes modulation of the incident laser pulse, resulting in additional scattering, thereby producing an instability. The transmission of laser light through a plasma, and the coupling of the laser energy into a plasma, can be greatly affected by Raman scattering, which consequently can have a large impact on various applications.

For example, Raman scattering in the forward direction (i.e., the scattered light is copropagating with the incident light) can be used to drive the self-modulated laser wakefield accelerator (for a review, see Ref. 1), in which a long (compared to the plasma wavelength) laser pulse becomes modulated and produces a large amplitude plasma wave with phase velocity near the speed of light $v_{\varphi}=\omega / k \simeq c$. This plasma wave, with $10-100 \mathrm{GeV} / \mathrm{m}$ accelerating gradients having been demonstrated using present laser technology, can be used to accelerate charged particles to high energies ${ }^{6-9}$. In laser fusion applications, such as the fast-ignitor ${ }^{4}$, the excitation of Raman instabilities can yield poor coupling of the laser to the energetic electrons. The use of finite-bandwidth laser pulses has been considered for enhancement or suppression of Raman instabilities ${ }^{10,11}$, and therefore as a means to control Raman scattering in these applications.

Several ultra-intense laser facilities around the world have been investigating the effect of frequency-chirped (i.e., frequency correlated to longitudinal position within the pulse) laser pulses propagating in an underdense plasma ${ }^{12-14}$. Experimental evidence by Faure et $a l .{ }^{12}$ has shown that the growth of the Raman instabilities is independent of the frequency chirp. Other experiments by Yau et al. ${ }^{13}$ have reported enhanced efficiency of the Raman 
forward scattering instability for positively-chirped laser pulses, and recent experiments by Leemans et al. ${ }^{14}$ reported frequency chirp induced asymmetries in the self-modulated laser wakefield electron yield. In addition, two-dimensional particle-in-cell (PIC) computer simulations presented in Ref. 15 claim enhancement of Raman forward scattering instabilities for positively-chirped laser pulses, however, these simulations assumed a large bandwidth $(20 \%)$, an order of magnitude beyond that used in present-day experiments ${ }^{12-14}$.

In this paper, we analyze the Raman forward scattering (RFS) of a short frequencychirped laser pulse of relativistic-intensity propagating in an underdense plasma, and calculate the effect of a correlated frequency chirp on the growth of the Raman instability using the coupled relativistic Maxwell-fluid equations. In Sec. II, we review the basic Maxwellfluid equations for the study of laser propagation in an underdense plasma. In Sec. III, the spatiotemporal growth of the plasma wave generated by RFS is calculated for a laser pulse with a linear frequency chirp in various spatiotemporal regimes. This includes a previously undescribed strongly-coupled four-wave nonresonant regime, which is important for relativistic laser intensities. It is shown that the growth rate increases (decreases) for positive (negative) chirp in all regimes of the RFS instability. The RFS growth rates are summarized in Sec. III F. In Sec. III G we examine the asymmetry between plasma wave generation using a laser pulse with positive and negative frequency chirp. It is shown that the effect of chirp on the growth rate is relatively minor, i.e., a few percent chirp yields few percent changes in the growth rates. In Sec. IV, numerical solutions of the full Maxwell-fluid equations are presented which confirm the analytic predictions. Section V presents a summary of the results, and discusses their relation to recent experiments. A calculation of chirped-pulse laser propagation in an underdense plasma neglecting the plasma response is presented in Appendix A. The dispersion relation for Raman scattering of an infinite homogeneous laser pulse is reviewed in Appendix B.

\section{BASIC FORMULATION AND ASSUMPTIONS}

In this section we describe the basic set of equations which govern the propagation of radiation in underdense plasmas. A one-dimensional (1D) model of the laser-plasma interaction is considered. A 1D model of the evolution of the laser pulse propagating in the plasma will be valid provided $k_{p}^{2} r_{s}^{2} \gg 1$, where $r_{s}$ is the laser spot size and $k_{p}=\omega_{p} / c$. The $1 \mathrm{D}$ fields 
associated with the pump laser, scattered light, and plasma response can be described by the transverse vector and the scalar potentials. In the Coulomb gauge, the Maxwell equations for the fields can be expressed in 1D as

$$
\begin{gathered}
{\left[\frac{\partial^{2}}{\partial z^{2}}-\frac{\partial^{2}}{\partial(c t)^{2}}\right] a=k_{p}^{2} \frac{n}{\gamma n_{0}} a,} \\
\frac{\partial^{2} \phi}{\partial z^{2}}=k_{p}^{2}\left(\frac{n}{n_{0}}-1\right),
\end{gathered}
$$

where $a$ and $\phi$ are the transverse vector and scalar potentials respectively normalized to $m c^{2} / e$. Note that, in the 1D approximation, conservation of transverse canonical momentum yields $\gamma \beta_{\perp}=a$ for an initially quiescent plasma, where $\gamma=\left(1-\beta_{z}^{2}-\beta_{\perp}^{2}\right)^{-1 / 2}$ is the relativistic Lorentz factor, and $\beta_{z}$ and $\beta_{\perp}$ are the electron longitudinal and transverse fluid velocities respectively normalized to the speed of light.

A cold-fluid model of the neutral plasma is assumed. Thermal effects may be ignored when the quiver velocity is much greater than the electron thermal velocity and the thermal energy spread is sufficiently small such that electron trapping in the plasma does not take place. The ions are also assumed to be stationary, which is typically the case for short-pulse $(\lesssim 1 \mathrm{ps})$ laser interactions in underdense plasmas. The cold fluid equations can be expressed in $1 \mathrm{D}$ as

$$
\begin{gathered}
\left(\frac{\partial}{\partial c t}+\beta_{z} \frac{\partial}{\partial z}\right)\left(\gamma \beta_{z}\right)=\frac{\partial}{\partial z} \phi-\frac{1}{2 \gamma} \frac{\partial}{\partial z} a^{2}, \\
\frac{\partial n}{\partial c t}+\frac{\partial}{\partial z}\left(n \beta_{z}\right)=0,
\end{gathered}
$$

where $n$ is the plasma number density.

To study the growth of Raman instabilities, we consider a density perturbation $\delta n=$ $n / n_{0}-1$, which results from the scattering of a large-amplitude pump laser pulse $a_{\text {pump }}$ into daughter waves $a_{\text {scat }}$, such that $\left|a_{\text {pump }}\right| \gg\left|a_{\text {scat }}\right|$. Linearizing about the perturbations $\delta n$ and $a_{\text {scat }}$, Eqs. (1)-(4) can be combined to yield

$$
\left[\frac{\partial^{2}}{\partial c^{2} t^{2}}+\frac{k_{p}^{2}}{\gamma_{\perp 0}}\right] \delta n=\frac{1}{\gamma_{\perp 0}^{2}} \frac{\partial^{2}}{\partial z^{2}}\left(a_{\text {pump }} a_{\text {scat }}\right)
$$

and

$$
\left[\frac{\partial^{2}}{\partial z^{2}}-\frac{\partial^{2}}{\partial c^{2} t^{2}}-\frac{k_{p}^{2}}{\gamma_{\perp 0}}\right] a_{\text {scat }}=\frac{k_{p}^{2}}{\gamma_{\perp 0}} a_{\text {pump }}\left[\delta n-\frac{1}{\gamma_{\perp 0}^{2}}\left(a_{\text {pump }} a_{\text {scat }}\right)\right],
$$

where $\gamma_{\perp 0}^{2}=1+\left\langle a_{\text {pump }}^{2}\right\rangle$, the angular brackets denote a time average over the fast laser period, and $k_{p} c / \gamma_{\perp 0}^{1 / 2}$ is the plasma frequency in the lab frame. It is convenient to work in 
the comoving variable $\zeta=z-c t$ and transform from the variables $(z, t)$ to $(\zeta=z-c t, \tau=c t)$. We will assume that the head of the right-going laser pulse is initially at $\zeta=0$ and the body of the laser pulse extends into the region $\zeta \leq 0$, while the plasma is unperturbed in the region $\zeta>0$. In the comoving variables $(\zeta, \tau)$ the linearized equations become

$$
\left[\left(\frac{\partial}{\partial \tau}-\frac{\partial}{\partial \zeta}\right)^{2}+\frac{k_{p}^{2}}{\gamma_{\perp 0}}\right] \delta n=\frac{1}{\gamma_{\perp 0}^{2}} \frac{\partial^{2}}{\partial \zeta^{2}}\left(a_{\text {pump }} a_{\text {scat }}\right)
$$

and

$$
\left[2 \frac{\partial^{2}}{\partial \zeta \partial \tau}-\frac{\partial^{2}}{\partial \tau^{2}}-\frac{k_{p}^{2}}{\gamma_{\perp 0}}\right] a_{\text {scat }}=\frac{k_{p}^{2}}{\gamma_{\perp 0}} a_{\text {pump }}\left[\delta n-\frac{1}{\gamma_{\perp 0}^{2}}\left(a_{\text {pump }} a_{\text {scat }}\right)\right] .
$$

We model the pump and scattered laser pulse normalized transverse vector potentials (linear polarization is assumed) as

$$
a=a_{\text {pump }}+a_{\text {scat }}=\frac{a_{0}}{2} e^{i \varphi_{0}}+\sum_{ \pm} \frac{a_{ \pm}}{2} e^{i \varphi_{ \pm}}+\text {c.c. }
$$

where $a_{0}$ is the pump amplitude, and $a_{ \pm}$are the slowly-varying envelopes of the Stokes (down-shifted) and anti-Stokes (up-shifted) scattered light waves. We will assume $\left|a_{0}\right| \gg$ $\left|a_{ \pm}\right|$and $a_{0}$ is a nonevolving envelope (i.e., pump depletion effects are neglected). To lowestorder, $\left\langle a^{2}\right\rangle \simeq\left\langle a_{\text {pump }}^{2}\right\rangle=\left|a_{0}\right|^{2} / 2$. The temporal and spatial derivatives of the phase determine the local values of the pulse frequency and wave number for the pump (subscript 0) and scattered (subscripts \pm ) light waves: $\omega_{0, \pm}=-\partial \varphi_{0, \pm} / \partial t$ and $k_{0, \pm}=\partial \varphi_{0, \pm} / \partial z$. The plasma density perturbation is modeled as

$$
\delta n=\frac{\hat{n}}{2} e^{i \varphi_{p}}+\text { c.c. }
$$

where $\hat{n}$ is the slowly-varying envelope of the plasma density perturbation and $\varphi_{p}=k z-\omega t$. The resonance condition for 1D Raman scattering requires $\varphi_{ \pm}=\varphi_{0} \pm \varphi_{p}$.

The effect of a finite-bandwidth on parametric instabilities, such as the Raman instability, has been extensively studied ${ }^{10,11}$ for the case of an uncorrelated, or random, frequency bandwidth. In this work we examine the effect of a correlated frequency chirp on the Raman instability growth rates. To isolate the effect of a correlated frequency chirp, we will consider laser pulses such that $k_{p} \sigma_{z} \gg 1$, where $\sigma_{z}$ is the root-mean square pulse length of the laser intensity, and the laser pulse vector potential amplitude is approximately uniform within the pulse. 
We consider a linear frequency chirp $\varphi_{0}=k_{0} \zeta+\epsilon \zeta^{2}$ on the pump laser pulse. If we also consider a Gaussian laser intensity distribution $a_{0}=\hat{a}_{0} \exp \left[-\zeta^{2} /\left(4 \sigma_{z}^{2}\right)\right]$, with constant frequency bandwidth $\sigma_{k}$ of the laser intensity, then the linear chirp, as a function of pulse duration and bandwidth, is $\epsilon=\left\{\left[\sigma_{k} /\left(2 \sigma_{z}\right)\right]^{2}-\left(2 \sigma_{z}\right)^{-4}\right\}^{1 / 2}$. Therefore the fullwidth-half-maximum (FWHM) relative chirp over a Gaussian pulse is $\Delta k_{e} / k_{0}=2 \epsilon L / k_{0}=$ $\sqrt{2 \ln 2}\left[\left(k_{0} \sigma_{0}\right)^{-2}-\left(k_{0} \sigma_{z}\right)^{-2}\right]^{1 / 2}$, where $\sigma_{0}$ is the Fourier transform-limited pulse length.

In this work we will focus our analysis on analytic solutions describing the RFS instability with the condition $k_{p}^{2} /\left(k_{0}^{2} \gamma_{\perp 0}\right) \ll 1$, i.e., laser propagation in an underdense plasma with group velocity of the laser approximately the speed of light. In addition, we note that highphase velocity plasma waves can also be generated by transverse Raman instabilities ${ }^{16,17}$; however, in this paper we will neglect these transverse effects and consider only direct RFS.

\section{RAMAN FORWARD SCATTERING}

In RFS, the scattered waves, which are referred to as the Stokes (down-shifted, $k_{-}=$ $k_{0}-k$ ) and anti-Stokes (up-shifted, $k_{+}=k_{0}+k$ ) waves, propagate in the same direction as the pump laser pulse $k_{ \pm} \sim k_{0} \gg k \sim k_{p}$. To solve Eqs. (7) and (8) for the growth of the RFS instability, it is convenient to define $\chi=\left(a_{0}^{*} a_{+}+a_{0} a_{-}^{*}\right) /\left(2 \gamma_{\perp 0}\right)-\gamma_{\perp 0} \hat{n}$. In the quasi-static approximation $^{18}, \chi$ is simply the amplitude of the plasma wave potential perturbation $\delta \phi=$ $\phi-\phi_{0}=(\chi / 2) \exp \left(i \varphi_{p}\right)+$ c.c., where $\phi_{0}=\gamma_{\perp 0}-1$ is the quasi-static equilibrium potential. Using the eikonal (slowly-varying amplitude) approximation $\left|\partial_{\tau} \chi\right| \ll|k \chi| \sim|\omega \chi| / c$ and $\left|\partial_{\tau} a_{ \pm}\right| \ll\left|k a_{ \pm}\right| \sim\left|\omega a_{ \pm}\right| / c$, Eq. (7) reduces to

$$
\left(\frac{\partial^{2}}{\partial \zeta^{2}}+2 i \frac{\omega}{c} \frac{\partial}{\partial \zeta}-D_{p}\right) \chi=\left[k^{2}-D_{p}-2 i\left(k-\frac{\omega}{c}\right) \frac{\partial}{\partial \zeta}\right] \frac{\left(a_{0}^{*} a_{+}+a_{0} a_{-}^{*}\right)}{2 \gamma_{\perp 0}},
$$

where $D_{p}=\omega^{2} / c^{2}-k_{p}^{2} / \gamma_{\perp 0}$ is the dispersion relation for the plasma wave. With the eikonal approximation $\left|\partial_{\zeta} a_{ \pm}\right| \ll\left|k_{ \pm} a_{ \pm}\right|$and $\left|\partial_{\tau} \varphi_{ \pm}\right| \ll\left|\partial_{\zeta} \varphi_{ \pm}\right|$, the evolution equations for the daughter waves Eq. (8) reduces to

$$
\begin{aligned}
{\left[2 i\left(\frac{\partial \varphi_{+}}{\partial \zeta}\right) \frac{\partial}{\partial \tau}+D_{+}\right] a_{+} } & =-\frac{k_{p}^{2}}{2 \gamma_{\perp 0}^{2}} a_{0} \chi, \\
{\left[-2 i\left(\frac{\partial \varphi_{-}}{\partial \zeta}\right) \frac{\partial}{\partial \tau}+D_{-}\right] a_{-}^{*} } & =-\frac{k_{p}^{2}}{2 \gamma_{\perp 0}^{2}} a_{0}^{*} \chi,
\end{aligned}
$$

where $D_{ \pm}=\omega_{ \pm}^{2} / c^{2}-k_{ \pm}^{2}-k_{p}^{2} / \gamma_{\perp 0}$ is the dispersion relation for each daughter wave. We take the pump wave to satisfy the usual dispersion relation $D_{0}=\omega_{0}^{2} / c^{2}-k_{0}^{2}-k_{p}^{2} / \gamma_{\perp 0}=0$. 
Note that, the resonance condition $\varphi_{ \pm}=\varphi_{0} \pm \varphi_{p}$ implies $\omega_{ \pm}=\omega_{0} \pm \omega$ and $k_{ \pm}=k_{0} \pm k$. The linear dispersion relation for the coupled equations Eqs. (11)-(13), assuming an infinite homogeneous unchirped pump laser pulse, is reviewed in Appendix B. Without loss of generality, we may take $D_{p}=0$ and $D_{-}=0$. With $D_{0}=0, D_{p}=0$, and $D_{-}=0$, the plasma wave number is $k^{2} \simeq k_{p}^{2} / \gamma_{\perp 0}$, the anti-Stokes wave is approximately resonant $\left|D_{+} / k_{0}^{2}\right| \simeq 2 k_{p}^{4} /\left(\gamma_{\perp 0}^{2} k_{0}^{4}\right) \ll 1$, and Eq. (11) reduces to

$$
\left(\frac{\partial^{2}}{\partial \zeta^{2}}+2 i k \frac{\partial}{\partial \zeta}\right) \chi=\frac{k^{2}}{2 \gamma_{\perp 0}}\left[a_{0}^{*} a_{+}+a_{0} a_{-}^{*}\right]
$$

For definiteness, in this work we will consider a pump laser pulse with a flat-top distribution such that $a_{0}(\zeta)=a_{0}$ for $\zeta \in[-L, 0]$ (i.e., the head of the pulse is located at $\zeta=0$ and the tail of the pulse at $\zeta=-L)$, and a linear chirp on the pump laser pulse $\varphi_{0}=k_{0} \zeta+\left(\Delta k_{e} / 2\right) \zeta(1+\zeta / L)$. The local wave number is $\partial_{\zeta} \varphi_{0}=k_{0}+\Delta k_{e}(\zeta / L+1 / 2)$, such that $k_{0}$ is the central wave number and $\Delta_{e} \equiv \Delta k_{e} / k_{0}$ is the relative chirp over the FWHM pump laser pulse length. By assuming this form of the pump laser pulse, we are neglecting pump dispersion effects. This is justified since the growth length of the Raman instabilities is much shorter than the dispersive broadening length, as discussed in Appendix A.

Several regimes of RFS can be identified ${ }^{19-21}$, and, as the instability grows, it passes through these various regimes depending on the value of $|\zeta| / \tau$ and the intensity of the incident laser pulse $a_{0}$. Past analytical analysis on Raman instabilities ${ }^{19-21}$ has primarily focused on nonrelativistic laser-plasma interactions where $a_{0} \ll 1$. In this work we perform a relativistic analysis of the linear RFS spatiotemporal growth rates including a correlated

frequency chirp. We also show that, for relativistic intensities $a_{0} \gtrsim 1$, the RFS instability can enter a strongly-coupled regime where the growth rate of the instability becomes larger than the plasma frequency.

\section{A. Four-wave resonant regime}

Consider the four-wave resonant interaction where both the Stokes and anti-Stokes modes are approximately resonant, and we will assume $D_{+} \simeq 0$ and $D_{-}=0$. We will also assume that we are in the weakly-coupled regime such that $\left|\partial_{\zeta} \chi\right| \ll|k \chi|$. Combining the envelope 
equations for plasma wave and scattered electromagnetic waves Eqs. (12)-(14) yields

$$
\begin{aligned}
\frac{\partial^{2} \chi}{\partial \zeta \partial \tau} & =\frac{k_{p}^{4} a_{0}^{*} a_{0}}{16 \gamma_{\perp 0}^{4} k}\left[\left(\frac{\partial \varphi_{+}}{\partial \zeta}\right)^{-1}-\left(\frac{\partial \varphi_{-}}{\partial \zeta}\right)^{-1}\right] \chi \\
& \simeq-\frac{k_{p}^{4}\left|a_{0}\right|^{2}}{8 \gamma_{\perp 0}^{4}}\left(\frac{\partial \varphi_{0}}{\partial \zeta}\right)^{-2} \chi=-\Gamma_{4 \text { loc }}^{2} \chi,
\end{aligned}
$$

where

$$
\Gamma_{4 \mathrm{loc}}(\zeta)=\frac{k_{p}^{2}\left|a_{0}\right|}{\sqrt{8} \gamma_{\perp 0}^{2} k_{\mathrm{loc}}}
$$

is the relativistic growth rate for the four-wave resonant RFS instability at the local wave number of the pump laser $k_{\text {loc }}(\zeta)=\partial_{\zeta} \varphi_{0}$. Applying the Laplace transform, $\mathcal{L}[\chi(\tau, \zeta)]=$ $\chi(\mathrm{s}, \zeta)$, to Eq. (15) yields

$$
\frac{\partial}{\partial \zeta}[s \chi(s, \zeta)-\chi(\tau=0, \zeta)]=-\Gamma_{4 \operatorname{loc}}^{2} \chi(s, \zeta)
$$

We will assume the initial condition $\partial_{\zeta} \chi(\tau=0, \zeta)=0$, i.e., the amplitude of the initial seed plasma wave perturbation is constant throughout the pump laser pulse. Consider a source of noise at the head of the pulse $\chi(\tau, \zeta=0)=\chi_{0} H(\tau)$, where $H$ is the Heaviside step function, such that $\mathcal{L}[\chi(\tau, \zeta=0)]=\chi(\mathrm{s}, \zeta=0)=\chi_{0} / \mathrm{s}$, i.e., the noise source at the front of the pulse is constant since the laser pulse is moving into fresh unperturbed plasma. Integrating Eq. (17) in $\zeta$ yields

$$
\chi(s, \zeta)=\frac{\chi_{0}}{s} \exp \left\{-\frac{\zeta \Gamma_{4 \mathrm{loc}}^{2}}{s} \frac{\left[1+\Delta_{e}\left(\frac{1}{2}+\frac{\zeta}{L}\right)\right]}{\left(1+\Delta_{e} / 2\right)}\right\} .
$$

Applying the inverse Laplace transform yields the solution for the amplitude of the plasma wave potential inside the laser pulse (for $-L \leq \zeta \leq 0$ )

$$
\chi(\tau, \zeta)=\chi_{0} H(\tau) I_{0}\left[2 \Gamma_{4 \mathrm{eff}} \sqrt{\tau|\zeta|}\right]
$$

where $I_{0}$ is the modified Bessel function of zeroth-order. Asymptotically $k_{p}^{2}|\zeta| \tau \gg 1$, the amplitude of the plasma wave grows exponentially. The effective growth rate of the plasma wave due to the four-wave resonant RFS instability is a function of position within the pump laser pulse:

$$
\Gamma_{4 \mathrm{eff}}(\zeta)=\Gamma_{4 \mathrm{loc}}\left[1-\Delta_{e} \frac{|\zeta|}{L}\left(1+\frac{\Delta_{e}}{2}\right)^{-1}\right]^{1 / 2} .
$$

For a positive chirp (i.e., $\Delta_{e}<0$, with red wavelengths at the head and blue wavelengths at the tail of the laser pulse), the RFS growth rate Eq. (20) is greater than the local growth 
rate throughout the laser pulse. Note that, for the unchirped case $\Delta_{e}=0$, the effective growth rate reduces to $\Gamma_{4 \mathrm{eff}}=\Gamma_{4}=k_{p}^{2}\left|a_{0}\right| /\left(\sqrt{8} \gamma_{\perp 0}^{2} k_{0}\right)$, the usual nonlinear growth rate for the four-wave resonant RFS instability ${ }^{22}$. For $\left|\Delta_{e}\right| \ll 1$, the lowest-order correction to the growth rate due to chirp is $\Gamma_{4 \mathrm{eff}} \simeq \Gamma_{4}\left[1-\left(\Delta_{e} / 2\right)(1-|\zeta| / L)\right]$.

Figure 1 shows the growth of the plasma wave amplitude $\chi / \chi_{0}$ due to the four-wave resonant RFS instability versus phase $k_{p} \zeta$ (location within the pump laser pulse) for the normalized parameters: $a_{0}=1, k_{0} / k_{p}=10$, and $k_{p} L=40$. The figure shows that for positive (negative) chirp $\Delta_{e}<0\left(\Delta_{e}>0\right)$, the growth rate of the four-wave resonant RFS instability is larger (smaller) than for the unchirped case $\left(\Delta_{e}=0\right)$.

As Eq. (19) indicates (and shown in Fig. 1), the growth of the instability is relatively insensitive to the frequency chirp. For example, at the center of the pulse, $\Gamma_{4 \text { eff }}(\zeta=-L / 2)=$ $\Gamma_{4}\left(1+\Delta_{e} / 2\right)^{-1 / 2}$, and the growth rate is changed by only $2.4 \%$ due to a $10 \%$ chirp over the pump laser pulse $\left(\Delta_{e}=0.1\right)$. Physically this can be understood through the resonance condition for the laser-plasma interaction $\varphi_{ \pm}=\varphi_{0} \pm \varphi_{p}$, which shows that the daughter waves (with group velocity $v_{g} \simeq c$ ) adopt approximately the local resonant frequency as determined by the local frequency of the pump laser $\omega_{ \pm}(\zeta)=\omega_{0}(\zeta) \pm k_{p} c / \gamma_{\perp 0}^{1 / 2}$. Therefore the resonant excitation of the instability continues as the plasma modulation (with group velocity $v_{g} \ll c$ ) slips through the pulse. The weak dependence on the sign of the chirp can be understood by considering the local growth rate. The growth of the instability at any location inside the pulse $\zeta$ will be determined by the plasma wave generation from the head to the location $\zeta$. Since the local growth rate [cf. Eq. (16)] is larger at the head for positive chirp (red wavelengths at the head), the enhancement in the growth rate for positive chirp is a result of larger cumulative growth of the plasma density modulation from the head of the pulse to $\zeta$, compared to negative chirp.

In deriving the four-wave resonant RFS growth rate it was assumed that $D_{-}=0$ (i.e., the Stokes wave is resonant) and $D_{+} \simeq 0$. Neglecting $D_{+}$in Eq. (12) requires $\left|2 k_{+} a_{+}^{-1} \partial_{\tau} a_{+}\right| \gg$ $\left|D_{+}\right|$. Therefore, the RFS instability will be in the four-wave resonant regime provided $\left|a_{0}\right|^{2}|\zeta| / \tau \gg 8\left(k_{p} / k_{0}\right)^{4}$. For sufficiently long times, this condition will no longer be satisfied, and the RFS instability will transition into the four-wave nonresonant regime. 


\section{B. Four-wave nonresonant regime}

In the weakly-coupled $\left(\left|\partial_{\zeta} \chi\right| \ll|k \chi|\right)$ four-wave nonresonant regime, Eqs. (12)-(14) can be combined to yield

$$
\left(\frac{\partial^{3}}{\partial \zeta \partial \tau^{2}}+\Gamma_{4 \operatorname{loc}}^{2} \frac{\partial}{\partial \tau}\right) \chi \simeq-i \Gamma_{N}^{3}\left[1+\Delta_{e}\left(\frac{1}{2}+\frac{\zeta}{L}\right)\right]^{-4} \chi,
$$

where we have kept the lowest-order term assuming $\left|D_{+} a_{+} /\left(2 k_{+} \partial_{\tau} a_{+}\right)\right|<1$. The relativistic temporal growth rate of the RFS in the four-wave nonresonant regime without chirp is

$$
\Gamma_{N}=\left(\frac{k_{p}^{7}\left|a_{0}\right|^{2}}{16 \gamma_{\perp 0}^{11 / 2} k_{0}^{4}}\right)^{1 / 3}
$$

We will assume that $\left|\Gamma_{4}^{2} \partial_{\tau} \chi\right| \ll\left|\Gamma_{N}^{3} \chi\right|$ such that the RFS interaction has moved from the four-wave resonant to the four-wave nonresonant regime and we may neglect the second term on the left-hand side of Eq. (21). Applying the Laplace transform to Eq. (21), and assuming the additional initial condition $\partial_{\tau} \chi(\tau=0, \zeta)=0$, yields

$$
\chi(s, \zeta)=\frac{\chi_{0}}{s} \exp \left(-\frac{\zeta \Gamma_{N}^{3}}{s^{2}} \frac{L}{3 \Delta_{e}}\left\{\left(1+\frac{\Delta_{e}}{2}\right)^{-3}-\left[1+\Delta_{e}\left(\frac{1}{2}+\frac{\zeta}{L}\right)\right]^{-3}\right\}\right) .
$$

The inverse Laplace transform can be evaluated asymptotically by using the method of steepest descents, and has the solution

$$
\chi(\tau, \zeta) \sim \chi_{0} \exp \left[\frac{3}{4}(\sqrt{3}+i) \Gamma_{N \text { eff }}\left(2|\zeta| \tau^{2}\right)^{1 / 3}\right],
$$

where the effective growth rate is

$$
\Gamma_{N \mathrm{eff}}=\Gamma_{N}\left(\frac{L}{3 \Delta_{e}|\zeta|}\right)^{1 / 3}\left\{-\left(1+\frac{\Delta_{e}}{2}\right)^{-3}+\left[1+\Delta_{e}\left(\frac{1}{2}+\frac{\zeta}{L}\right)\right]^{-3}\right\}^{1 / 3}
$$

For $\left|\Delta_{e}\right| \ll 1$, the lowest-order correction to the growth rate due to chirp is $\Gamma_{N \text { eff }} \simeq \Gamma_{N}[1-$ $\left.\left(2 \Delta_{e} / 3\right)(1-|\zeta| / L)\right]$

In deriving Eq. (24) it was assumed that $\left|\Gamma_{4}^{2} \partial_{\tau} \chi\right| \ll\left|\Gamma_{N}^{3} \chi\right|$. Using Eq. (24), this condition reduces to $|\zeta| / \tau \ll 2\left(\gamma_{\perp 0} / a_{0}^{2}\right)\left(k_{p} / k_{0}\right)^{2}$. Therefore, for sufficiently long times, the RFS will transition from the four-wave resonant regime $\left(\left|\Gamma_{4}^{2} \partial_{\tau} \chi\right| \gg\left|\Gamma_{N}^{3} \chi\right|\right)$ to the four-wave nonresonant regime $\left(\left|\Gamma_{4}^{2} \partial_{\tau} \chi\right| \ll\left|\Gamma_{N}^{3} \chi\right|\right)$. For longer times, the RFS instability can transition, for $a_{0} \ll 1$, into the three-wave regime where the anti-Stokes wave can be neglected, or, for $a_{0} \gtrsim 1$, into the strongly-coupled four-wave nonresonant regime. 


\section{Three-wave regime}

In the three-wave regime, we assume that the anti-Stokes wave is sufficiently out of resonance such that $\left|D_{+} a_{+}\right| \gg\left|2 k_{+} \partial_{\tau} a_{+}\right|$. In this regime the anti-Stokes term may be neglected, and Eqs. (13) and (14) can be combined to yield

$$
\frac{\partial^{2} \chi}{\partial \zeta \partial \tau} \simeq-\Gamma_{3}^{2}\left[1+\Delta_{e}\left(\frac{1}{2}+\frac{\zeta}{L}\right)\right]^{-1} \chi
$$

where

$$
\Gamma_{3}=\frac{k_{p}^{3 / 2}\left|a_{0}\right|}{4 \gamma_{\perp 0}^{7 / 4} k_{0}^{1 / 2}}
$$

is the relativistic temporal growth rate of the RFS instability in the three-wave regime without chirp. The solution to Eq. (26) (for $-L \leq \zeta \leq 0)$ is

$$
\chi(\tau, \zeta)=\chi_{0} H(\tau) I_{0}\left[2 \Gamma_{3 \mathrm{eff}} \sqrt{\tau|\zeta|}\right],
$$

where the effective growth rate of RFS in the three-wave regime is

$$
\Gamma_{3 \mathrm{eff}}=\Gamma_{3}\left\{\frac{L}{\zeta \Delta_{e}} \ln \left[1+\Delta_{e} \frac{\zeta}{L}\left(1+\frac{\Delta_{e}}{2}\right)^{-1}\right]\right\}^{1 / 2} .
$$

For $\left|\Delta_{e}\right| \ll 1$, the lowest-order correction to the growth rate due to chirp is $\Gamma_{3 \mathrm{eff}} \simeq \Gamma_{3}[1-$ $\left.\left(\Delta_{e} / 4\right)(1-|\zeta| / L)\right]$.

In deriving Eq. (26), the anti-Stokes wave was neglected $\left|D_{+} a_{+}\right| \gg\left|2 k_{+} \partial_{\tau} a_{+}\right|$. Using Eq. (28) this condition for the three-wave RFS regime yields $|\zeta| / \tau \ll 16 /\left(a_{0}^{2} \gamma_{\perp 0}^{1 / 2}\right)\left(k_{p} / k_{0}\right)^{5}$. In addition, the weakly-coupled approximation $\left|\partial_{\zeta} \chi\right| \ll|k \chi|$, assumed in Secs. III A-III C,

will no longer be valid at long times for sufficiently intense laser pules $a_{0} \gtrsim 1$. Assuming the perturbation grows in the resonant four-wave regime, the weakly-coupled approximation implies $|\zeta| / \tau \gg\left(k_{p} / k_{0}\right)^{2} a_{0}^{2} /\left(8 \gamma_{\perp 0}^{3}\right)$. For intense laser pulses at long times the RFS will violate this condition and transition into the strongly-coupled regime.

\section{Strongly-coupled four-wave nonresonant regime}

In the strongly-coupled $\left|\partial_{\zeta} \chi\right| \gg|k \chi|$ four-wave nonresonant $\left|\Gamma_{4}^{2} \partial_{\tau} \chi\right| \ll\left|\Gamma_{N}^{3} \chi\right|$ regime, Eqs. (12)-(14) can be combined to yield

$$
\frac{\partial^{4} \chi}{\partial \zeta^{2} \partial \tau^{2}} \simeq 2 k \Gamma_{N}^{3}\left[1+\Delta_{e}\left(\frac{1}{2}+\frac{\zeta}{L}\right)\right]^{-4} \chi
$$


for an flat-top pump laser pulse with a linear frequency chirp. The eikonal approximation $\left|\partial_{\tau} \chi\right| \ll|k \chi|$ is still assumed since the RFS instability enters the strongly-coupled fourwave nonresonant regime in the asymptotic limit $k_{p} \tau \gg 1$. The solution to Eq. (30) in the asymptotic limit can be approximated as

$$
\begin{aligned}
\chi \sim \chi_{0} \exp \left[2^{5 / 4}\left(k \Gamma_{N}^{3}\right)^{1 / 4} \tau^{1 / 2}\left(\frac{L}{\Delta_{e}}\right)^{1 / 2}\right. & \\
& \left.\times\left\{\left[1+\Delta_{e}\left(\frac{1}{2}+\frac{\zeta}{L}\right)\right]^{-1}-\left(1+\frac{\Delta_{e}}{2}\right)^{-1}\right\}^{1 / 2}\right] .
\end{aligned}
$$

For small chirp $\left|\Delta_{e}\right| \ll 1$, the RFS instability in the strongly-coupled four-wave nonresonant regime has the exponentiation $|\chi| \sim\left|\chi_{0}\right| \exp \left(N_{4 \mathrm{sc}}\right)$, with

$$
N_{4 \mathrm{sc}} \simeq 2^{5 / 4}\left(k \Gamma_{N}^{3}\right)^{1 / 4}(|\zeta| \tau)^{1 / 2}\left[1-\frac{\Delta_{e}}{2}\left(1-\frac{|\zeta|}{L}\right)\right] .
$$

The RFS instability will be in the strongly-coupled four-wave nonresonant regime provided $16\left(k_{p} / k_{0}\right)^{5} /\left(a_{0}^{2} \gamma_{\perp 0}^{1 / 2}\right) \ll|\zeta| / \tau \ll\left(k_{p} / k_{0}\right)^{2} a_{0}^{2} /\left(8 \gamma_{\perp 0}^{3}\right)$. This condition will be violated for sufficiently large pump laser intensities and propagation times, and the RFS instability will transition into the strongly-coupled three-wave regime.

\section{E. Strongly-coupled three-wave regime}

In the asymptotic limit $k_{p} \tau \gg 1$, the RFS instability will enter the strongly-coupled three-wave regime. In the asymptotic strongly-coupled three-wave regime, $\left|\partial_{\zeta} \chi\right| \gg|k \chi|$, $\left|\partial_{\tau} \chi\right| \ll|k \chi|$, and the anti-Stokes wave is no longer in resonance; therefore Eqs. (13) and (14) can be combined to yield

$$
\frac{\partial^{3} \chi}{\partial \zeta^{2} \partial \tau} \simeq-2 i k \Gamma_{3}^{2}\left[1+\Delta_{e}\left(\frac{1}{2}+\frac{\zeta}{L}\right)\right]^{-1} \chi,
$$

for an flat-top pump laser pulse with a linear frequency chirp. The asymptotic solution to Eq. (33) can be approximated as

$$
\begin{aligned}
\chi \sim \chi_{0} \exp \left[\frac{3}{2^{2 / 3}}(\sqrt{3}-i)\left(k \Gamma_{3}^{2}\right)^{1 / 3}\right. & \tau^{1 / 3}\left(\frac{L}{\Delta_{e}}\right)^{2 / 3} \\
& \left.\times\left\{\left(1+\frac{\Delta_{e}}{2}\right)^{1 / 2}-\left[1+\Delta_{e}\left(\frac{1}{2}+\frac{\zeta}{L}\right)\right]^{1 / 2}\right\}^{2 / 3}\right] .
\end{aligned}
$$


For small chirp $\left|\Delta_{e}\right| \ll 1$, the RFS instability in the strongly-coupled three-wave regime has the exponentiation $|\chi| \sim\left|\chi_{0}\right| \exp \left(N_{3 \mathrm{sc}}\right)$, with

$$
N_{3 \mathrm{sc}} \simeq \frac{3^{3 / 2}}{2^{2 / 3}}\left(k \Gamma_{3}^{2}\right)^{1 / 3}\left(|\zeta|^{2} \tau\right)^{1 / 3}\left[1-\frac{\Delta_{e}}{6}\left(1-\frac{|\zeta|}{L}\right)\right] .
$$

\section{F. Summary of RFS instability regimes}

Table I summarizes the exponentiation (number of e-folds) of the RFS instability for a laser pulse with linear frequency $\operatorname{chirp}\left(\partial_{\zeta}^{2} \varphi_{0}=k_{0} \Delta_{e} / L\right)$, for $\left|\Delta_{e}\right| \ll 1$, in the regimes: four-wave resonant $\left(N_{4}\right)$, four-wave nonresonant $\left(N_{4 \mathrm{nr}}\right)$, three-wave $\left(N_{3}\right)$, strongly-coupled four-wave nonresonant $\left(N_{4 \mathrm{sc}}\right)$, and strongly-coupled three-wave $\left(N_{3 \mathrm{sc}}\right)$. In each regime the exponentiation is increased for positive chirp (red wavelengths at the head of the pulse, blue wavelengths at the tail, such that $\left.\Delta_{e}<0\right)$. Physically this is due to the enhanced local growth of the plasma density modulations at the head of the pulse for positive chirp, compared to negative chirp, in all RFS instability regimes. The enhanced plasma density modulations will slip through the pulse seeding the instability and producing larger growth of the Raman instability throughout the pulse. We also note that, in the limit of no chirp $\Delta_{e}=0$ and for weakly-relativistic laser intensities $a_{0}<1$, the four-wave resonant, four-wave nonresonant, three-wave, and strongly-coupled three-wave regime growth rates reduce to essentially those described previously ${ }^{20,21}$. However, the strongly-coupled four-wave nonresonant regime is a new regime of RFS, which has not been previously analyzed. This new regime is only important for relativistic laser intensities.

Figure 2 shows schematically the regimes of the FRS instability in $\left(a_{0},|\zeta| / \tau\right)$ parameter space for $k_{0} / k_{p}=10$. Initially the instability is dominated by the four-wave resonant mode (region 1 of Fig. 2). As the propagation time increases, the instability transitions into the four-wave nonresonant regime (region 2 of Fig. 2). Note that the instability always enters the four-wave nonresonant regime before moving into the strongly-coupled regime. At later propagation times, the instability will transition into the three-wave regime (region 3 of Fig. 2) for nonrelativistic interactions $a_{0} \ll 1$, or the strongly-coupled four-wave nonresonant regime (region 4 of Fig. 2) for relativistic interactions $\left[a_{0}^{4} / \gamma_{\perp 0}^{5 / 2}>2^{7}\left(k_{p} / k_{0}\right)^{3}\right]$. For sufficiently long times, provided the interaction has not become nonlinear, the RFS instability will transition into the strongly-coupled three-wave regime (region 5 of Fig. 2).

In this work a 1D model was assumed. The 1D approximation will be valid for laser pulse 
evolution if the transverse Laplacian $\nabla_{\perp}^{2}$ in the wave equation operator can be neglected compared to the evolution operator for the scattered daughter waves $2 k_{ \pm} \partial_{\tau}$ [cf. the lefthand side of Eq. (12)]. If we assume that the transverse gradient scales as the laser spot size $\nabla_{\perp} \sim 1 / r_{s}$, then the $1 \mathrm{D}$ limit $\left|\nabla_{\perp}^{2} a_{ \pm}\right| \ll\left|2 k_{ \pm} \partial_{\tau} a_{ \pm}\right|$is valid provided $1 /\left(k_{p} r_{s}\right)^{4} \ll$ $\left[a_{0}^{2} /\left(2 \gamma_{\perp 0}^{4}\right)\right]|\zeta| / \tau$ in the four-wave resonant regime. This condition can be rewritten as $1 \ll$ $\left(4 \sqrt{2} / \gamma_{\perp 0}^{2}\right)\left[\left(P / P_{c}\right)\left(k_{p} / k_{0}\right)\left(k_{p}|\zeta|\right)\left(Z_{R} / \tau\right)\right]^{1 / 2}$, where $Z_{R}=k_{0} r_{s}^{2} / 2$ is the laser Rayleigh length and $P_{c}=k_{p}^{2} a_{0}^{2} r_{s}^{2} / 32$ is the critical power for relativistic self-guiding. As may be expected, for typical laser-plasma parameters (e.g., $P / P_{c} \sim 1, k_{0} / k_{p} \sim 10$, and $k_{p} L \sim 10-100$ ), the 1D model will no longer be valid for propagation distances longer than a Rayleigh length $\tau>Z_{R}$

As mentioned in Sec. II, high-phase velocity plasma waves can also be generated by the two-dimensional Raman sidescatter instability. The influence of chirp on Raman sidescatter can be studied by including a transverse wavenumber $k_{\perp}$ in the analysis of Sec. III. It can be shown that Raman sidescatter can be neglected provided $\left(k_{\perp} / k_{p}\right)^{4} \ll\left[a_{0}^{2} /\left(4 \gamma_{\perp 0}^{5}\right)\right]\left(k_{p} / k_{0}\right)^{2}|\zeta| / \tau$. The influence of chirp on Raman sidescatter and the self-modulation instability will be the subject of a future publication.

\section{G. RFS chirp asymmetry}

As Table I indicates, the spatiotemporal growth rate in all regimes of RFS is larger for a positively-chirped $\left(\Delta_{e}<0\right)$ than for a negatively-chirped $\left(\Delta_{e}>0\right)$ laser pulse. We may examine the asymmetry between laser pulses with positive and negative chirp by considering the ratio of the energy in the RFS generated plasma wave for the chirped to unchirped pump laser pulse

$$
\frac{\mathcal{E}\left(\tau ; \Delta_{\mathrm{e}}\right)}{\mathcal{E}(\tau ; 0)}=\frac{\int_{L}\left|\chi\left(\Delta_{e}\right)\right|^{2} d \zeta}{\int_{L}|\chi(0)|^{2} d \zeta}
$$

Figure 3(a) shows the plasma wave energy excited in the four-wave resonant regime by a laser pulse with a linear frequency chirp normalized to the unchirped excitation energy Eq. (36) versus propagation time $k_{p} \tau$, for $a_{0}=1, k_{0} / k_{p}=10$, and $k_{p} L=40$. Figure 3(b) shows the ratio of the plasma wave energy excited by positive and negative chirped pulses $\mathcal{E}\left(-\left|\Delta_{e}\right|\right) / \mathcal{E}\left(\left|\Delta_{e}\right|\right)$ versus the magnitude chirp $\left|\Delta_{e}\right|$ after propagating $k_{p} \tau=100,200$, and 300 , for $a_{0}=1, k_{0} / k_{p}=10$, and $k_{p} L=40$. Figure 3 illustrates the relatively small influence of the frequency chirp on plasma wave generation. Physically the relatively weak 
dependence of chirp on the instability growth is due to the scattered daughter waves adopting approximately the local resonant frequency as determined by the local frequency of the pump laser pulse. Therefore the resonant excitation is maintained throughout the pulse. These results are also consistent with recent experimental observations, as discussed in Sec. V.

\section{NUMERICAL RESULTS}

To verify the analytical work of Sec. III, the nonlinear 1D cold Maxwell-fluid equations were solved numerically. The details of the numerical methods used are discussed in Refs. 23 and 24. The numerical solution is carried out in terms of the cold fluid momentum equation, driven by the Lorentz force, together with the continuity equation for the evolution of the plasma density and the Maxwell equations, coupled to the plasma current and charge density. The numerical model retains the fast time-scale of the laser field. The plasma ions are assumed stationary. No approximations, beyond the hydrodynamic assumptions are introduced. For these examples, the equations were discretized using second-order methods in both space and time. One of the significant advantages of partial differential equation based models, especially when studying instabilities, is their inherent lack of "numerical" noise. While the numerical solution is subject to various errors, including round-off, associated with the unavoidable discretization of the underlying equations, these errors are manifest as smooth alterations to the solution. There is no intrinsic source of "random" noise to contaminate the solution. This allows for precise (reproducible) control over the seed for the instability.

The numerically calculated growth of the RFS instability is plotted in Figs. 4 and 5 . In the numerical solutions, the laser pulse was initialized with a constant transverse vector potential amplitude (flat-top longitudinal distribution with a smoothed head and tail) and a linear frequency chirp. The laser pulse, initially in vacuum, propagates up a density ramp [with scale length $k_{p} n /(d n / d \zeta) \simeq 50$ ], which smoothly transitions into a uniform plasma. The pulse was initialized outside the plasma to ensure self-consistent fluid and field initial conditions. The propagation time was taken to be zero when the head of the pump laser pulse reaches the center of the plasma density ramp.

Figure 4 shows the numerically calculated normalized longitudinal electric field $E_{z} / E_{0}$, where $E_{0}=k_{p} m c^{2} / e$, versus phase $k_{p} \zeta$ for both positive chirp $\Delta_{e}=-0.2$ (solid curve) 
and negative chirp $\Delta_{e}=0.2$ (dashed curve) after the propagation time $k_{p} \tau=550$ for the parameters $a_{0}=0.5, k_{p} L=60$, and $k_{0} / k_{p}=10$. The fast density oscillations (at $2 k_{0}$ ), due to the electron quiver motion in the laser field, as well as the oscillations at the plasma frequency, are clearly visible in the numerical solution shown in Fig. 4. The analytically calculated amplitude $\chi$ (in the four-wave resonant regime) of the plasma wave is also plotted in Fig. 4 for $\Delta_{e}=-0.2$ (dashed-dotted curve) and $\Delta_{e}=0.2$ (dotted curve). The initial seed of the analytic solution $\chi_{0}$ was chosen to correspond to the first peak of the plasma oscillation in the numerical solution. In Fig. 4, good agreement in the growth of the plasma wave amplitude versus distance behind the head $\zeta$ is observed between the analytic RFS growth calculation $\left[\chi\right.$ from Eq. (19)] and the peaks of the numerically calculated $E_{z} / E_{0}$ curves.

In Fig. 5, the normalized longitudinal electric field $E_{z} / E_{z}(0)$, calculated from numerical integration of the nonlinear 1D cold-fluid equations, is plotted versus propagation time $k_{p} \tau$ at a fixed location behind the head of the pump laser pulse $\left(\left|k_{p} \zeta\right|=32\right.$, with pulse

length $\left.k_{p} L=60\right)$ for positive $\left(\Delta_{e}=-0.2\right)$ and negative $\left(\Delta_{e}=0.2\right)$ chirped laser pulses with the parameters $a_{0}=0.5$ and $k_{0} / k_{p}=10$. The numerical calculation is compared to the analytically calculated growth of the plasma wave amplitude $\left[\chi / \chi_{0}\right.$ from Eq. (19)]. The numerical solution includes the full nonlinear group velocity and pump laser evolution effects, such as pump depletion. Good agreement is observed in Fig. 5 between analytically and numerically calculated RFS growth. Agreement begins to breakdown in Fig. 5 between the theoretical and numerically calculated curves for large propagation time as the RFS instability begins to transition into the four-wave nonresonant regime.

\section{DISCUSSION AND SUMMARY}

In this work, we have presented a calculation of the spatiotemporal growth of the Raman forward scattering instability produced by a frequency-chirped laser pulse propagating in an underdense plasma using the relativistic Maxwell-fluid equations. It was shown that a frequency chirp correlated to the longitudinal position within the laser pulse alters the exponentiation of the RFS instability. Table I summarizes the RFS growth rates in various regimes of the RFS instability. In particular, it was shown that positive chirped pulses (red wavelengths at the head of the pulse and blue wavelengths at the tail of the pulse) have 
a larger growth rate than negative chirped pulses (blue wavelengths at the head and red wavelengths at the tail).

The enhanced growth of the instability for positive chirp is due to the enhanced axial energy transport at the head of the pulse resulting from the larger local growth rate (for red wavelengths). This enhanced axial energy transport at the head produces enhanced modulation of the plasma density. Since the group velocity of the plasma wave is very low $\left(v_{g} \ll c\right)$, it slips back through the pulse, seeding the Raman instability. The scattered daughter light waves adopt the local frequency $\omega_{ \pm}(\zeta)=\omega_{0}(\zeta) \pm k_{p} c / \gamma_{\perp 0}^{1 / 2}$. Since both the daughter and pump waves have group velocities $v_{g} \simeq c$, resonance is maintained. This continues to drive the plasma density modulation, resulting in larger growth of the instability for positive chirp throughout the pulse.

The RFS instability is initially seeded by plasma density fluctuations or pump laser intensity fluctuations which contain Fourier components at the relativistic plasma frequency $k=k_{p} / \gamma_{\perp 0}^{1 / 2}$. For example the seeding of the RFS may be generated by thermal fluctuations in the plasma, ionization-induced plasma waves (owing to a time-varying dielectric) ${ }^{25-27}$, or ponderomotively-excited plasma waves (owing to the laser intensity gradient) ${ }^{28,29}$. Experiments often will use the same pump laser pulse which undergoes self-modulation to create the plasma through ionization of a gas. Typically photoionization will occur very early in the head of the laser pulse, where the laser electric field becomes sufficiently intense such that the rate of ionization is maximum ${ }^{30}$. This will create a plasma density front moving with the laser. The amplitude of the ionization-induced plasma waves created by this ionization front will be approximately $\chi_{0} \sim a_{0}^{2}\left(\zeta_{\text {ionz }}\right) / 4$, where $\zeta_{\text {ionz }}$ is the location in the pump laser pulse where the laser electric field is sufficiently intense to ionize the background gas. Note that the ionization location $\zeta_{\text {ionz }}$ is weakly dependent on the chirp. For typical laser experimental parameters, the shift in the ionization location is less than a laser wavelength $\zeta_{\text {ionz }}<\lambda_{0}$. The amplitude of the ponderomotively-excited plasma waves scales as $\chi_{0} \sim a_{0}^{2} /\left(k_{p} L\right)^{2}$, where the gradient in the laser pulse intensity is $\sim 1 / L$. For short laser pulses, the ponderomotively excited plasma wave will typically dominate other sources for seeding the RFS instability.

Previous theoretical work by Dodd and Umstadter ${ }^{15}$ on Raman scattering of a chirped laser pulse used a heuristic calculation of the group velocity dispersion (GVD) to estimate the effect of a linear frequency chirp. Dodd and Umstadter claimed that, in the nonrelativistic regime $\left(a_{0} \ll 1\right)$, the amount of chirp necessary to eliminate the Raman scattering instability 
is $\Delta \omega_{0}=\left(\omega_{0} / \omega_{p}\right) \gamma_{\mathrm{R}} / 2$ where $\gamma_{\mathrm{R}}=\left(\omega_{p} / \sqrt{8}\right)\left(\omega_{p} / \omega_{0}\right) a_{0}$ is the usual unchirped temporal growth rate. This predicts a complete stabilization using a bandwidth of $\Delta \omega_{0} / \omega_{0} \simeq 1.8 \%$ for the parameters $a_{0}=1$ and $\omega_{0} / \omega_{p}=10$. Furthermore PIC simulations of Dodd and Umstadter imply stabilization of RFS using a $20 \%$ bandwidth for the parameters $a_{0}=1$ and $\omega_{0} / \omega_{p}=10$. In contrast, the analytic results presented in Sec. III, as well as numerical solutions to the nonlinear 1D equations, show only a modest change in the RFS growth rate. We find, for a $20 \%$ negative chirp $\left(\Delta_{e}=0.2\right)$, the growth rate in the four-wave resonant regime at the center of the pulse is reduced by only $4.7 \%$ compared to the unchirped growth rate.

A linear chirp will affect the GVD, which can expand (for negative chirp) or contract (for positive chirp) the pulse. The change in group velocity due to the frequency chirp is approximately $\Delta \beta_{g} \simeq\left(\omega_{p} / \omega_{0}\right)^{2} \Delta \omega_{0} / \omega_{0}$. The propagation distance $L_{\mathrm{GVD}}$ required for the laser pulse to bunch at the plasma wavelength (which will drive or suppress the RFS growth) due to a linear chirp over a plasma wavelength $\Delta \omega_{0} / \omega_{0}=\Delta_{e} \lambda_{p} / L$ can be estimated as $L_{\mathrm{GVD}} \simeq \lambda_{p} / \Delta \beta_{g}$. For typical laser-plasma parameters (e.g., $a_{0} \sim 1, k_{0} / k_{p} \sim 10$, and $k_{p} L \sim 10-100$ ), the propagation distance for GVD to drive or suppress the RFS growth is much greater than the growth length for the RFS instability, i.e., $k_{p} L_{\mathrm{GVD}} \simeq\left(\omega_{0} / \omega_{p}\right)^{2}\left(k_{p} L\right) / \Delta_{e} \gg \omega_{p} \gamma_{\mathrm{R}}^{-1}=\sqrt{8}\left(\omega_{0} / \omega_{p}\right) / a_{0}$. This indicates that, although the chirp affects the GVD throughout the pulse, the enhanced bunching due to GVD has an insignificant effect on the RFS process.

Recent experiments ${ }^{12,13}$ using long laser pulses $\left(k_{p} L \gg 1\right)$ with a frequency chirp $\left|\Delta_{e}\right| \approx 3 \%$ operating in the self-modulated laser wakefield regime have measured RFS growth independent of the chirp. These experiments are consistent with the calculation presented in Sec. III where a $3 \%$ chirp produces little asymmetry [cf. Sec. III G]. Experiments operating in the regime $k_{p} L \gtrsim 1$, i.e., the standard laser wakefield accelerator regime, have reported chirp-induced asymmetry in the measured Stokes wave ${ }^{13}$ and electron yield ${ }^{14}$. We believe this asymmetry in the standard laser wakefield regime is due to preferential seeding of the RFS instability by asymmetric pulse envelopes generated by nonlinear contributions to the chirp in the laser compression system ${ }^{14}$.

To conclude, in this paper we have examined the Raman forward scattering of a frequencychirped short-pulse laser pulse of relativistic-intensity in an underdense plasma. The spatiotemporal growth of the plasma wave was calculated using the relativistic Maxwell-fluid equations for a laser pulse with a linear frequency chirp in various regimes. A new regime 
of RFS was identified, the strongly-coupled four-wave nonresonant regime, which is important for relativistic laser intensities. It was shown that the growth rate of RFS increases (decreases) for positive (negative) chirp. In addition, we have shown that a linear frequency chirp with $\left|\Delta_{e}\right| \ll 1$ produces only a small effect on the growth of the RFS instabilities, and therefore will have only a minor effect on the enhancement or suppression of Raman instabilities for laser-plasma applications such as laser-driven plasma wakefield accelerators, laser-plasma-based radiation sources, and fast-ignitor fusion schemes.

\section{Acknowledgments}

This work was supported by the U.S. Department of Energy under Contract No. DEAC03-76SF0098.

\section{APPENDIX A: CHIRPED PULSE PROPAGATION}

In this appendix we consider the propagation of a frequency-chirped low-intensity ( $a_{0} \ll$ 1) laser pulse in an underdense plasma. In the nonrelativistic regime $a_{0}^{2} \ll 1$ we may neglect the plasma response $\partial_{\tau} \chi \simeq 0$. The $1 \mathrm{D}$ linear wave equation for a laser pulse propagating in a homogeneous plasma, in the comoving variables $(\zeta, \tau)$, is

$$
\left[\frac{\partial^{2}}{\partial \tau^{2}}-2 \frac{\partial^{2}}{\partial \zeta \partial \tau}+k_{p}^{2}\right] a=0 .
$$

Consider $a=\hat{a} \exp \left(i k_{0} \zeta\right)$, where $\hat{a}$ is a slowly-varying envelope. Substituting into the wave equation Eq. (A1) yields

$$
\left[\frac{\partial^{2}}{\partial \tau^{2}}-2\left(i k_{0}+\frac{\partial}{\partial \zeta}\right) \frac{\partial}{\partial \tau}+k_{p}^{2}\right] \hat{a}=0 .
$$

The term $\partial^{2} \hat{a} / \partial \tau^{2}$ may be neglected for short laser pulses propagating in underdense plasmas. Applying the Fourier transform $\mathcal{F}[\hat{a}(\zeta, \tau)]=\hat{a}(\delta k, \tau)=\hat{a}_{k}(\tau)$ yields the solution

$$
\hat{a}_{k}=\hat{a}_{k}(0) \exp \left[\frac{-i k_{p}^{2} \tau}{2\left(k_{0}+\delta k\right)}\right] .
$$

Consider, as an initial condition, a laser pulse with a Gaussian axial profile and a linear chirp:

$$
\hat{a}(\zeta, \tau=0)=a_{0} \exp \left[-\left(1-i \epsilon L^{2}\right) \zeta^{2} / L^{2}\right],
$$


where $L$ is the pulse length, and $\epsilon$ is the chirp parameter. The Fourier transform of the initial condition Eq. (A4) is

$$
\hat{a}_{k}(0)=\frac{a_{0}}{\sqrt{2}} \frac{L}{\left(1+\epsilon^{2} L^{4}\right)^{1 / 4}} \exp \left[\frac{i}{2} \tan ^{-1}\left(\epsilon L^{2}\right)-\frac{(\delta k)^{2} L^{2}}{4} \frac{\left(1+i \epsilon L^{2}\right)}{\left(1+\epsilon^{2} L^{4}\right)}\right] .
$$

We will assume that $|\delta k| \sim 1 / L \ll k_{0}$ and retain only second-order dispersive effects. With this approximation, $k_{0} /\left(k_{0}+\delta k\right) \simeq 1-\delta k / k_{0}+(\delta k)^{2} / k_{0}^{2}$, and the inverse Fourier transform of Eq. (A3) yields the solution

$$
a=a_{0}\left(\frac{L}{L_{e}}\right)^{1 / 2} \exp \left[i k_{0}\left(\zeta-\frac{k_{p}^{2} \tau}{2 k_{0}^{2}}\right)+\frac{i}{2}\left(\tan ^{-1} \epsilon L^{2}-\tan ^{-1} \eta\right)-\frac{\zeta^{\prime 2}}{L_{e}^{2}}(1-i \eta)\right]
$$

where

$$
L_{e}=L\left(\frac{1+\eta^{2}}{1+\epsilon^{2} L^{4}}\right)^{1 / 2}
$$

is the effective pulse length, $\eta=\epsilon L^{2}+\left(1+\epsilon^{2} L^{4}\right) \tau / Z_{D}$, and $Z_{D}=k_{0}^{3} L^{2} /\left(2 k_{p}^{2}\right)$ is the characteristic length for dispersive spreading ${ }^{31}$. The pulse envelope $\hat{a}$ propagates at $\zeta^{\prime}=z-\beta_{g} c t=\zeta+\tau k_{p}^{2} /\left(2 k_{0}^{2}\right)$, where the normalized group velocity is $\beta_{g}=1-k_{p}^{2} /\left(2 k_{0}^{2}\right)$. Note that $\zeta-\tau k_{p}^{2} /\left(2 k_{0}^{2}\right)=z-\beta_{\varphi} c t$, where the normalized phase velocity is $\beta_{\varphi}=1+k_{p}^{2} /\left(2 k_{0}^{2}\right)$, such that the dispersion relation $\omega_{0}^{2} / c^{2}=k_{0}^{2}+k_{p}^{2}$ is approximately satisfied and $\beta_{g} \beta_{\varphi} \simeq 1$. The effective wave number of the pulse is $k_{e}=k_{0}+2 \eta \zeta^{\prime} / L_{e}^{2}$.

Dispersion will cause broadening of the pulse length. A frequency chirp will modify the pulse broadening, as indicated in Eq. (A7). A positive chirp $(\epsilon<0)$ will have red wavelengths in the front of the pulse and blue wavelengths in the back of the pulse, such that the group velocity is larger in the back than the front, resulting in pulse compression. Figure 6 shows the effective pulse length $L_{e} / L$ versus propagation time $\tau / Z_{D}$ for a laser pulse with positive chirp $\epsilon L^{2}=-0.5$, negative chirp $\epsilon L^{2}=0.5$, and zero chirp $\epsilon=0$. As the figure shows, pulse compression occurs for a positively-chirped pulse for propagation times $\tau / Z_{D}<2|\epsilon| L^{2} /\left(1+\epsilon^{2} L^{4}\right)$, with the minimum pulse length of $L_{e}=1 /\left(\epsilon^{2} L^{3}\right)$ at the propagation time $\tau / Z_{D}=1 /\left(|\epsilon| L^{2}\right)$.

For short propagation distances $\tau \ll Z_{D}$ the dispersive broadening of the pulse may be neglected, and the pump laser evolves as

$$
a=a_{0} \exp \left[i k_{0}\left(\zeta-\frac{\tau k_{p}^{2}}{2 k_{0}^{2}}\right)\right] \exp \left[-\frac{\zeta^{\prime 2}}{L^{2}}\left(1-i \epsilon L^{2}\right)\right],
$$

where the effective wave number is $k_{e}=k_{0}+2 \epsilon \zeta^{\prime}$. The characteristic length for dispersive spreading is typically much longer than the growth length for Raman instabilities, i.e., 
$k_{p} Z_{D}=\left(k_{p} L\right)^{2}\left(k_{0} / k_{p}\right)^{3} / 2 \gg k_{p} \Gamma_{4}^{-1}=\sqrt{8} \gamma_{\perp 0}^{2}\left(k_{0} / k_{p}\right) /\left|a_{0}\right|$ for $a_{0} \sim 1, k_{0} / k_{p} \gg 1$, and $k_{p} L \sim 1$ (i.e., we may consider the rate at which a single modulation of length $\sim \lambda_{p}$ disperses). In Sec. III we have neglected dispersive broadening and assumed a flat-top intensity distribution propagating in an underdense plasma well below the critical density, such that $a_{\text {pump }} \simeq\left(a_{0} / 2\right) \exp \left[i \varphi_{0}(\zeta)\right]+$ c.c., where $\varphi_{0}=k_{0} \zeta+\left(\Delta k_{e} / 2\right) \zeta(1+\zeta / L)$.

\section{APPENDIX B: DISPERSION RELATION}

In this appendix we review the dispersion relation for 1D Raman scattering of an infinite homogeneous laser. Equations (11)-(13) describe the coupling of the plasma wave and scattered light waves. In the infinite homogeneous pump limit, Eqs. (11)-(13) may be combined to yield the following dispersion relation for Raman scattering

$$
\frac{D_{p}}{k^{2}-D_{p}}=\frac{k_{p}^{2}\left|a_{0}\right|^{2}}{4 \gamma_{\perp 0}^{3}}\left(\frac{1}{D_{-}}+\frac{1}{D_{+}}\right) .
$$

Here $D_{p}=\omega^{2} / c^{2}-k_{p}^{2} / \gamma_{\perp 0}$ is the dispersion relation for the plasma wave, and $D_{ \pm}=\omega_{ \pm}^{2} / c^{2}-$ $k_{ \pm}^{2}-k_{p}^{2} / \gamma_{\perp 0}$ is the dispersion relation for each scattered wave, with $\omega_{ \pm}=\omega_{0} \pm \omega$ and $k_{ \pm}=k_{0} \pm k$. The pump laser pulse satisfies the equilibrium dispersion relation $D_{0}=$ $\omega_{0}^{2} / c^{2}-k_{0}^{2}-k_{p}^{2} / \gamma_{\perp 0}=0$. The dispersion relation for Raman scattering Eq. (B1) can be used to calculate the purely temporal growth rates in the long pulse limit (i.e., infinite homogeneous pump laser pulse without chirp $)^{5}$. Equation (B1) is valid for a relativistic pump laser pulse and, in the limit $a_{0} \ll 1$, reduces to the well-known weakly-relativistic dispersion relation.

In the weakly-coupled regime, the frequency of the plasma wave is approximately the plasma frequency $\omega=k_{p} c / \gamma_{\perp 0}+\delta \omega$, where $\delta \omega \ll \omega$, and the dispersion relation for the plasma wave is $c^{2} D_{p} \simeq-2 k_{p} c \delta \omega / \gamma_{\perp 0}$. The plasma wave number $k \simeq k_{p} / \gamma_{\perp 0}^{1 / 2}$ is determined from the resonance conditions $D_{-}=0$ and $D_{0}=0$.

In the four-wave regime, $c^{2} D_{ \pm} \simeq-2\left(k_{p} c / \gamma_{\perp 0} \pm \omega_{0}\right) \delta \omega$. Substituting into the Raman dispersion relation Eq. (B1), yields the temporal growth rate in the four-wave resonant regime $\gamma_{4}=\operatorname{Im}(\delta \omega)=k_{p}^{2} c^{2}\left|a_{0}\right| /\left(\sqrt{8} \omega_{0} \gamma_{\perp 0}\right)$.

In the four-wave nonresonant regime, $c^{2} D_{-} \simeq 2\left(\omega_{0}-k_{p} c / \gamma_{\perp 0}\right) \delta \omega$ and $D_{+} \simeq$ $-2 k_{p}^{4} c^{2} /\left(\gamma_{\perp 0}^{2} \omega_{0}^{2}\right)$. Substituting into the Raman dispersion relation Eq. (B1), yields the temporal growth rate in the four-wave nonresonant regime $\gamma_{4 \mathrm{nr}}=\operatorname{Im}(\delta \omega)=$ 
$\left(\sqrt{3} / 2^{7 / 3}\right)\left|a_{0}\right|^{2 / 3}\left(k_{p} c / \omega_{0}\right)^{4 / 3} k_{p} c / \gamma_{\perp 0}^{11 / 6}$.

In the three-wave regime $\left|D_{+}\right| \gg\left|D_{-}\right|$and $c^{2} D_{-} \simeq 2 \omega_{0} \delta \omega$. The 1D Raman dispersion relation Eq. (B1), yields the temporal growth rate $\gamma_{3}=\operatorname{Im}(\delta \omega)=\left(k_{p} c\right)^{3 / 2}\left|a_{0}\right| /\left(4 \gamma_{\perp 0}^{7 / 4} \omega_{0}^{1 / 2}\right)$ in the three-wave regime.

In the strongly-coupled regime, the growth rate is much greater than the plasma frequency, therefore $\omega \simeq \delta \omega$ with $\delta \omega \gg k_{p} c / \gamma_{\perp 0}^{1 / 2}$, and the dispersion relation for the plasma wave is $c^{2} D_{p} \simeq \delta \omega^{2}$.

In the strongly-coupled four-wave nonresonant regime, $c^{2} D_{-} \simeq-2 \omega_{0} \delta \omega$ and $D_{+} \simeq$ $2 \omega_{0} \delta \omega-2 k_{p}^{4} c^{2} /\left(\gamma_{\perp 0}^{2} \omega_{0}^{2}\right)$. Substituting into the Raman dispersion relation Eq. (B1), yields the temporal growth rate in the strongly-coupled four-wave nonresonant regime $\gamma_{4 \mathrm{sc}}=\operatorname{Im}(\delta \omega)=$ $2^{-3 / 4}\left|a_{0}\right|^{1 / 2}\left(k_{p} c\right)^{2} /\left(\omega_{0} \gamma_{\perp 0}^{3 / 2}\right)$.

In the three-wave regime $\left|D_{+}\right| \gg\left|D_{-}\right|$and $c^{2} D_{-} \simeq-2 \omega_{0} \delta \omega$. The $1 \mathrm{D}$ Raman dispersion relation Eq. (B1) yields the temporal growth rate $\gamma_{3 s c}=\operatorname{Im}(\delta \omega)=$ $(\sqrt{3} / 4) k_{p} c\left(k_{p} c / \omega_{0}\right)^{1 / 3}\left|a_{0}\right|^{2 / 3} k_{p} c / \gamma_{\perp 0}^{4 / 3}$ in the strongly-coupled three-wave regime.

Comparing the temporal growth rates derived from the dispersion relation in the infinite homogeneous pump limit Eq. (B1) with the spatiotemporal growth rates derived in Sec. III, one finds the same scaling with the parameters $a_{0}, k_{p}$, and $k_{0}$ in each regime of the RFS instability.

The dispersion relation Eq. (B1) also contains the physics of Raman backscatter (RBS). For direct RBS, only the counterpropagating Stokes wave is resonant, and the resonant condition $\varphi_{0}=\varphi_{-}+\varphi_{p}$ implies $k \simeq 2 k_{0}$ and $D_{-} \simeq-2 \omega_{0} \omega$. The dispersion relation Eq. (B1) can be expressed for RBS as

$$
\omega\left(\omega^{2}+k_{p}^{2} c^{2} \gamma_{\perp 0}^{-1}\right)=\frac{k_{p}^{2} c^{2}\left|a_{0}\right|^{2} \omega_{0}}{2 \gamma_{\perp 0}^{3}}=\omega_{c}^{3},
$$

where $\omega_{c}$ is the strength of coupling between the backscattered light and the density perturbation. In the weakly-coupled or Raman regime ${ }^{5}, \omega_{c} \ll k_{c} c / \gamma_{\perp 0}^{1 / 2}$, which will be satisfied provided $a_{0} \ll 1$, and the dispersion relation yields the temporal growth rate $\operatorname{Im}(\omega)=$ $\left(\omega_{0} k_{p} c\right)^{1 / 2}\left|a_{0}\right| / 2$. In the strongly-coupled or Compton regime ${ }^{20}, \omega_{c} \gg k_{c} c / \gamma_{\perp 0}^{1 / 2}$, and the dispersion relation yields the temporal growth rate $\operatorname{Im}(\omega)=\sqrt{3} / 2^{4 / 3}\left(a_{0}^{2 / 3} / \gamma_{\perp 0}\right)\left(k_{p}^{2} c^{2} \omega_{0}^{2}\right)^{1 / 3}$. 
1 E. Esarey, P. Sprangle, J. Krall, and A. Ting, IEEE Trans. Plasma Sci. 24, 252 (1996).

2 E. Esarey, A. Ting, P. Sprangle, D. Umstadter, and X. Liu, IEEE Trans. Plasma Sci. 21, 95 (1993).

3 P. Amendt, D. C. Eder, and S. C. Wilks, Phys. Rev. Lett. 66, 2589 (1991).

4 M. Tabak, J. Hammer, M. E. Glinsky, W. L. Kruer, S. C. Wilks, J. Woodworth, E. M. Campbell, M. D. Perry, and R. J. Mason, Phys. Plasmas 1, 1626 (1994).

5 W. L. Kruer, The Physics of Laser Plasma Interactions (Addison-Wesley, Redwood City, CA, 1988).

6 A. Modena, Z. Najmudin, A. E. Dangor, C. E. Clayton, K. A. Marsh, C. Joshi, V. Malka, C. B. Darrow, C. Danson, D. Neely, et al., Nature 377, 606 (1995).

7 D. Umstadter, S.-Y. Chen, A. Maksimchuk, G. Mourou, and R. Wagner, Science 273, 472 (1996).

8 A. Ting, C. I. Moore, K. Krushelnick, C. Manka, E. Esarey, P. Sprangle, R. Hubbard, H. R. Burris, R. Fischer, and M. Baine, Phys. Plasmas 4, 1889 (1997).

9 W. P. Leemans, D. Rodgers, P. E. Catravas, C. G. R. Geddes, G. Fubiani, E. Esarey, B. A. Shadwick, R. Donahue, and A. Smith, Phys. Plasmas 8, 2510 (2001).

10 G. Laval, R. Pellat, D. Pesme, A. Ramani, M. N. Rosenbluth, and E. A. Williams, Phys. Fluids 20, 2049 (1977).

11 P. N. Guzdar, C. S. Liu, and R. H. Lehmberg, Phys. Fluids B 3, 2882 (1991).

12 J. Faure, J.-R. Marques, V. Malka, F. Amiranoff, Z. Najmudin, B. Walton, J.-P. Rousseau, S. Ranc, A. Solodov, and P. Mora, Phys. Rev. E 63, 065401 (2001).

13 T.-W. Yau, C.-J. Hsu, H.-H. Chu, Y.-H. Chen, C.-H. Lee, J. Wang, and S.-Y. Chen, Phys. Plasmas 9, 391 (2002).

14 W. P. Leemans, P. Catravas, E. Esarey, C. G. R. Geddes, C. Toth, R. Trines, C. B. Schroeder, B. A. Shadwick, J. van Tilborg, and J. Faure, Phys. Rev. Lett. 89, 174802 (2002).

15 E. S. Dodd and D. Umstadter, Phys. Plasma 8, 3531 (2001).

16 E. Esarey, J. Krall, and P. Sprangle, Phys. Rev. Lett. 72, 2887 (1994).

17 N. E. Andreev, V. I. Kirsanov, L. M. Gorbunov, and A. S. Sakharov, IEEE Trans. Plasma Sci. 24, 363 (1996). 
18 P. Sprangle, E. Esarey, and A. Ting, Phys. Rev. Lett. 64, 2011 (1990).

19 T. M. Antonsen, Jr. and P. Mora, Phys. Rev. Lett. 69, 2204 (1992).

20 T. M. Antonsen, Jr. and P. Mora, Phys. Fluids B 5, 1440 (1993).

21 C. D. Decker, W. B. Mori, T. Katsouleas, and D. E. Hinkel, Phys. Plasmas 3, 1360 (1996).

22 W. B. Mori, C. D. Decker, D. E. Hinkel, and T. Katsouleas, Phys. Rev. Lett. 72, 1482 (1994).

23 B. A. Shadwick, G. M. Tarkenton, E. H. Esarey, and W. P. Leemans, IEEE Trans. Plasma Sci. 30, 38 (2002).

24 B. A. Shadwick, G. M. Tarkenton, E. H. Esarey, and W. P. Leemans, Proceedings of the 9th Advanced Accelerator Concepts Workshop, Santa Fe, 2000, edited by P. L. Colestock and S. Kelley (Amer. Inst. Phys., NY, 2001), AIP Conf. Proc. Vol. 279, p. 477.

25 W. B. Mori and T. Katsouleas, Phys. Rev. Lett. 69, 3495 (1992).

26 D. L. Fisher, T. Tajima, and M. C. Downer, Proceedings of the 5th Advanced Accelerator Concepts Workshop, Port Jefferson, 1992, edited by J. S. Wurtele (Amer. Inst. Phys., NY, 1993), AIP Conf. Proc. Vol. 279, p. 477.

27 D. L. Fisher and T. Tajima, Phys. Rev. E 53, 1844 (1996).

28 P. Sprangle, E. Esarey, A. Ting, and G. Joyce, Appl. Phys. Lett. 53, 2146 (1988).

29 J. Krall, E. Esarey, P. Sprangle, and G. Joyce, Phys. Plasmas 1, 1738 (1994).

30 P. Sprangle, E. Esarey, and J. Krall, Phys. Rev. E 54, 4211 (1996).

31 E. Esarey and W. P. Leemans, Phys. Rev. E 59, 1082 (1999). 
TABLE I: Raman forward scattering growth rates (number of $e$-folds) in the four-wave resonant $\left(N_{4}\right)$, four-wave nonresonant $\left(N_{4 \mathrm{nr}}\right)$, three-wave $\left(N_{3}\right)$, strongly-coupled four-wave nonresonant $\left(N_{4 \mathrm{sc}}\right)$, and strongly-coupled three-wave $\left(N_{3 \mathrm{sc}}\right)$ regimes, including frequency chirp for $\left|\Delta_{e}\right| \ll 1$.

\begin{tabular}{|c|c|}
\hline Exponentiation & Regime : \\
\hline$N_{4} \simeq \frac{1}{2^{1 / 2}} \frac{a_{0}}{\gamma_{\perp 0}^{2}}\left(\frac{k_{p}}{k_{0}}\right) k_{p}(|\zeta| \tau)^{1 / 2}\left[1-\frac{\Delta_{e}}{2}\left(1-\frac{|\zeta|}{L}\right)\right]$ & $2 \frac{\gamma_{\perp 0}}{a_{0}^{2}}\left(\frac{k_{p}}{k_{0}}\right)^{2} \ll \frac{|\zeta|}{\tau}$ \\
\hline$N_{4 \mathrm{nr}} \simeq \frac{3^{3 / 2}}{8} \frac{a_{0}^{2 / 3}}{\gamma_{\perp 0}^{11 / 6}}\left(\frac{k_{p}}{k_{0}}\right)^{4 / 3} k_{p}\left(|\zeta| \tau^{2}\right)^{1 / 3}\left[1-\frac{2 \Delta_{e}}{3}\left(1-\frac{|\zeta|}{L}\right)\right]$ & $\frac{16}{a_{0}^{2} \gamma_{\perp 0}^{1 / 2}} \frac{k_{p}^{5}}{k_{0}^{5}} \ll \frac{|\zeta|}{\tau} \ll 2 \frac{\gamma_{\perp 0}}{a_{0}^{2}} \frac{k_{p}^{2}}{k_{0}^{2}}$ and $\frac{|\zeta|}{\tau} \gg \frac{a_{0}^{2}}{8 \gamma_{\perp 0}^{3}} \frac{k_{p}^{2}}{k_{0}^{2}}$ \\
\hline$N_{3} \simeq \frac{1}{2} \frac{a_{0}}{\gamma_{\perp 0}^{7 / 4}}\left(\frac{k_{p}}{k_{0}}\right)^{1 / 2} k_{p}(|\zeta| \tau)^{1 / 2}\left[1-\frac{\Delta_{e}}{4}\left(1-\frac{|\zeta|}{L}\right)\right]$ & $\frac{a_{0}^{2}}{8 \gamma_{\perp 0}^{3}}\left(\frac{k_{p}}{k_{0}}\right)^{2} \ll \frac{|\zeta|}{\tau} \ll \frac{16}{a_{0}^{2} \gamma_{\perp 0}^{1 / 2}}\left(\frac{k_{p}}{k_{0}}\right)^{5}$ \\
\hline$N_{4 \mathrm{sc}} \simeq \frac{1}{2^{1 / 4}} \frac{a_{0}^{1 / 2}}{\gamma_{\perp 0}^{3 / 2}}\left(\frac{k_{p}}{k_{0}}\right) k_{p}(|\zeta| \tau)^{1 / 2}\left[1-\frac{\Delta_{e}}{2}\left(1-\frac{|\zeta|}{L}\right)\right]$ & $\frac{16}{a_{0}^{2} \gamma_{\perp 0}^{1 / 2}}\left(\frac{k_{p}}{k_{0}}\right)^{5} \ll \frac{|\zeta|}{\tau} \ll \frac{a_{0}^{2}}{8 \gamma_{\perp 0}^{3}}\left(\frac{k_{p}}{k_{0}}\right)^{2}$ \\
\hline$N_{3 \mathrm{sc}} \simeq \frac{3^{3 / 2}}{4} \frac{a_{0}^{2 / 3}}{\gamma_{\perp 0}^{4 / 3}}\left(\frac{k_{p}}{k_{0}}\right)^{1 / 3} k_{p}\left(\zeta^{2} \tau\right)^{1 / 3}\left[1-\frac{\Delta_{e}}{6}\left(1-\frac{|\zeta|}{L}\right)\right]$ & $\frac{|\zeta|}{\tau} \ll \frac{16}{a_{0}^{2} \gamma_{\perp 0}^{1 / 2}}\left(\frac{k_{p}}{k_{0}}\right)^{5}$ and $\frac{|\zeta|}{\tau} \ll \frac{a_{0}^{2}}{8 \gamma_{\perp 0}^{3}}\left(\frac{k_{p}}{k_{0}}\right)^{2}$ \\
\hline
\end{tabular}


FIG. 1: Growth of plasma wave potential $\chi / \chi_{0}$ due to RFS versus phase $k_{p} \zeta$ at three times $k_{p} \tau=100, k_{p} \tau=200$, and $k_{p} \tau=300$ for the parameters: $a_{0}=1, k_{0} / k_{p}=10$, and $k_{p} L=40$. Solid line is laser pulse without chirp $\Delta_{e}=0$, dashed line is laser pulse with negative chirp $\Delta_{e}=0.1$, and dotted line is laser pulse with positive chirp $\Delta_{e}=-0.1$.

FIG. 2: Regimes of RFS instability [in parameter space $\left(a_{0},|\zeta| / \tau\right)$ for $k_{0} / k_{p}=10$ ]: (1) four-wave resonant, (2) four-wave nonresonant, (3) three-wave, (4) strongly-coupled four-wave nonresonant, and (5) strongly-coupled three-wave.

FIG. 3: (a) The plasma wave energy excited by a frequency chirped laser pulse normalized to the unchirped excitation energy versus propagation time $k_{p} \tau$ for several chirps $\Delta_{e}=-0.1,-0.05,0,0.05$, and 0.1 , with $a_{0}=1, k_{0} / k_{p}=10$ and $k_{p} L=40$. (b) The ratio of the plasma wave energy excited by positive and negative chirped pulses $\mathcal{E}\left(-\left|\Delta_{e}\right|\right) / \mathcal{E}\left(\left|\Delta_{e}\right|\right)$ versus chirp $\left|\Delta_{e}\right|$ after $k_{p} \tau=100,200$, and 300, with $a_{0}=1, k_{0} / k_{p}=10$, and $k_{p} L=40$.

FIG. 4: Numerically calculated longitudinal electric field $E_{z} / E_{0}$ for $\Delta_{e}=-0.2$ (solid curve) and $\Delta_{e}=0.2$ (dashed curve) versus distance behind the head of the pump laser pulse $k_{p} \zeta$ for $a_{0}=0.5$ and $k_{0} / k_{p}=10$. Also shown is the theoretically calculated growth of the plasma wave amplitude $\chi$ for $\Delta_{e}=-0.2$ (dashed-dotted curve) and $\Delta_{e}=0.2$ (dotted curve).

FIG. 5: Numerically calculated normalized longitudinal electric field $E_{z} / E_{z}(0)$ (dotted curves) and theoretically calculated plasma wave amplitude $\chi / \chi_{0}$ (solid curves) plotted versus propagation time $k_{p} \tau$ at fixed distance behind the head of the pump laser pulse for positive $\left(\Delta_{e}=-0.2\right)$ and negative $\left(\Delta_{e}=0.2\right)$ chirp, with $a_{0}=0.5$ and $k_{0} / k_{p}=10$.

FIG. 6: Effective pulse length $L_{e} / L$ versus propagation time $\tau / Z_{D}$ for a laser pulse with positive $\epsilon L^{2}=-0.5$, negative $\epsilon L^{2}=0.5$, and zero $\epsilon=0$ chirp. 


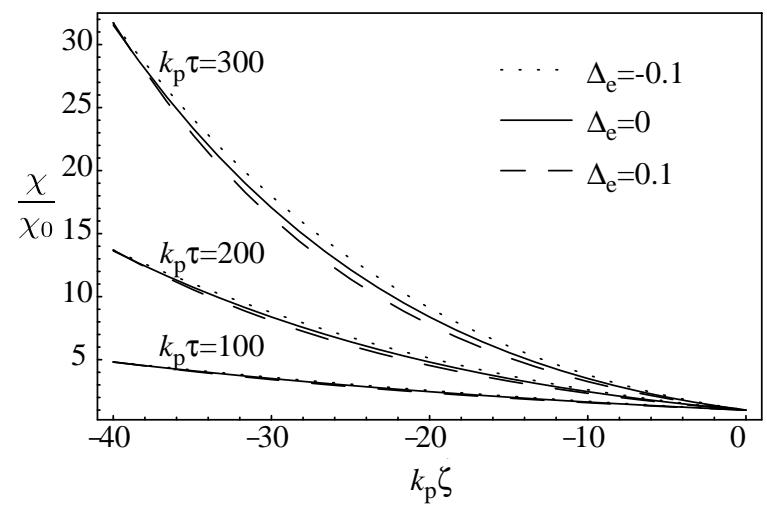

Schroeder et al., Fig. 1 


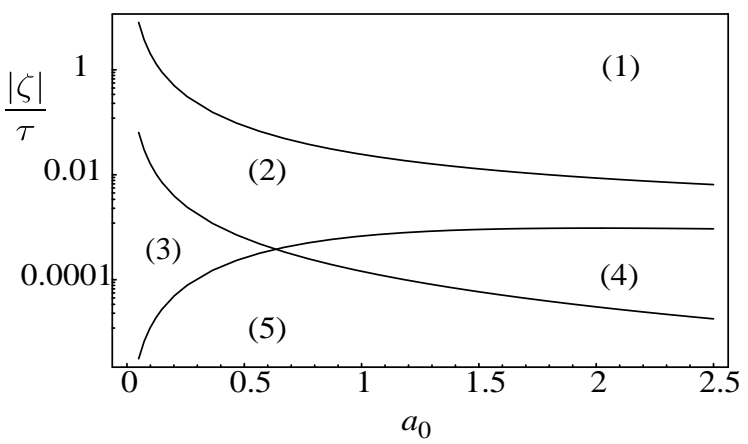

Schroeder et al., Fig. 2 

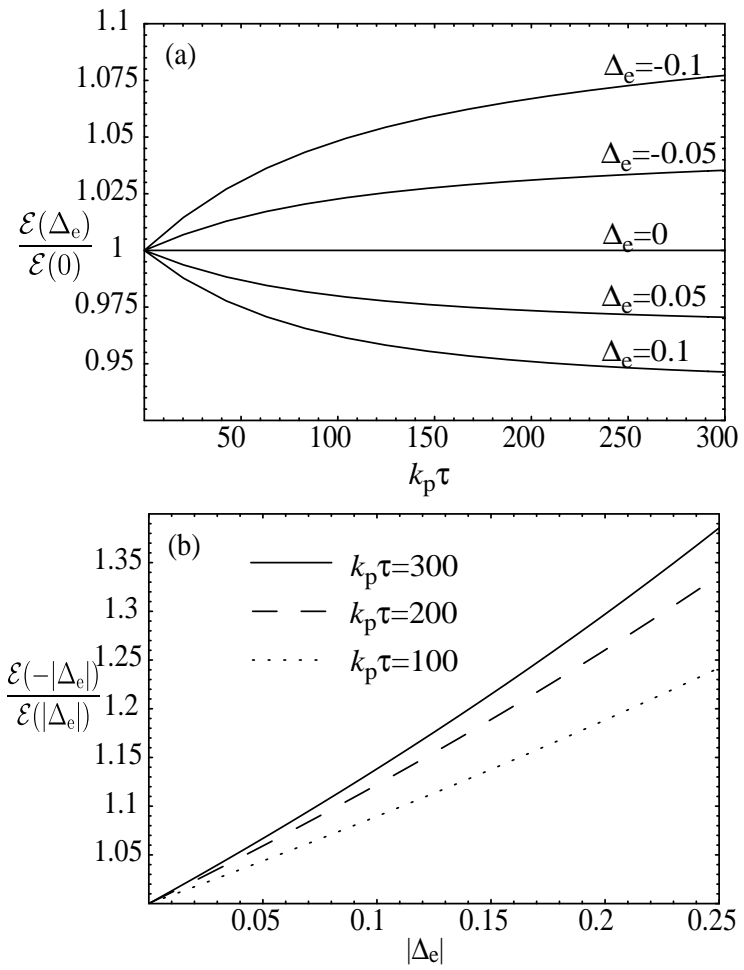

Schroeder et al., Fig. 3 


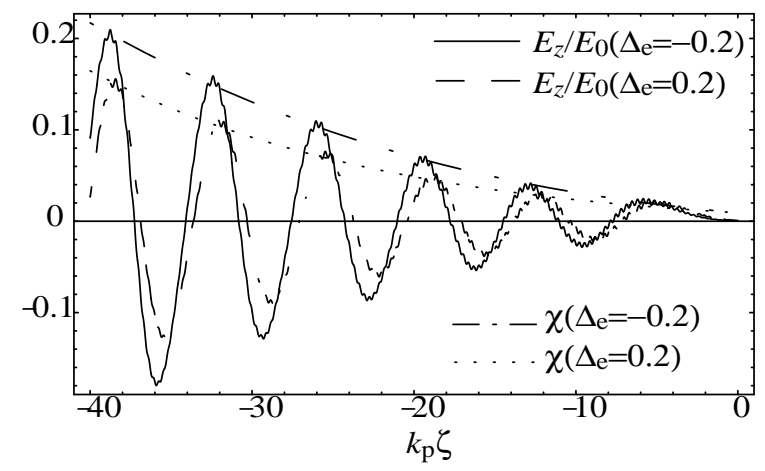

Schroeder et al., Fig. 4 


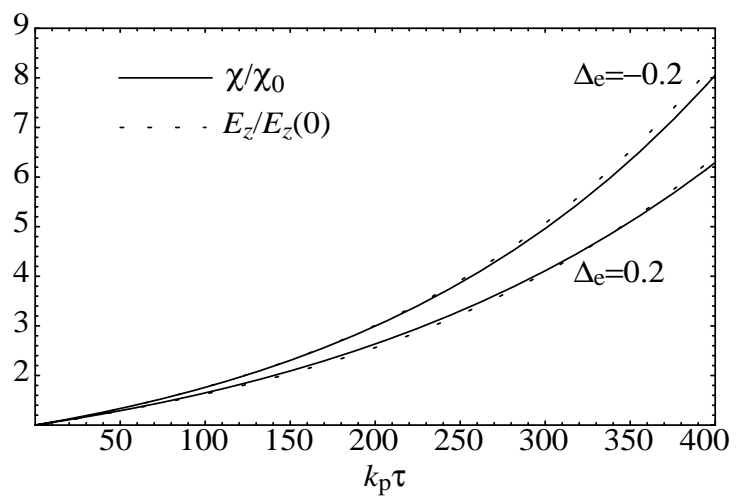

Schroeder et al., Fig. 5 


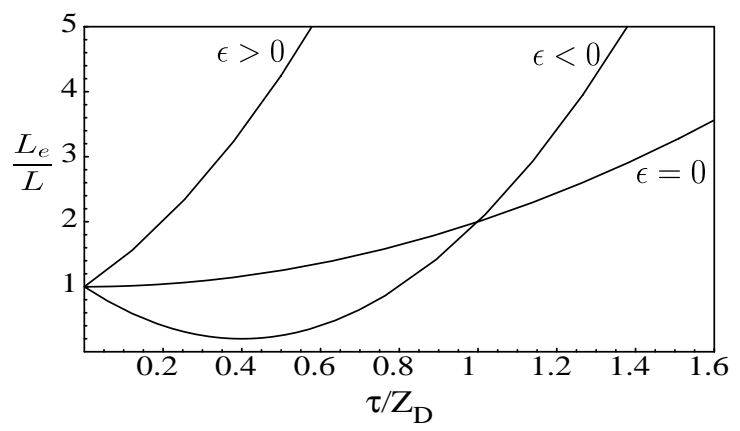

Schroeder et al., Fig. 6 